\title{
Cardiovascular Benefits of Plant-Based Diets
}

\author{
Marcella Rodrigues Guedes ${ }^{1}$ and Márcia Regina Simas Torres Klein ${ }^{1}$ (D) \\ Universidade do Estado do Rio de Janeiro (UERJ), Rio de Janeiro, RJ - Brazil \\ Editorial referring to the article: Better Adequacy of Food Intake According to Dietary Recommendations of National Cholesterol Education \\ Program in Vegetarian Compared to Omnivorous Men
}

Increasing evidence suggests that plant-based dietary patterns, characterized by higher intake of plant foods and lower intake of animal foods, confer benefits to cardiovascular health. ${ }^{1-4}$ Vegetarian diets are a subset of plant-based diets that exclude some or all animal products (vegan diets). ${ }^{5}$

A meta-analysis of 86 cross-sectional and 10 cohort prospective studies evaluated the association between vegetarian, vegan diets, risk factors for chronic diseases, risk of all-cause mortality, incidence, and mortality from cardio-cerebrovascular diseases. The overall analysis of cross-sectional studies revealed significantly reduced levels of body mass index, total cholesterol, LDL-cholesterol, and glucose levels in vegetarians and vegans compared with omnivores. In relation to cohort studies, the analysis showed a significantly reduced risk of incidence and/or mortality from ischemic heart disease by $25 \%{ }^{6}$

Yokoyama et al., ${ }^{7}$ conducted a meta-analysis of 30 observational studies and 19 clinical trials to assess the association of plant-based diets and plasma lipids. The authors concluded that plant-based diets are associated with decreased total cholesterol, LDL-cholesterol, and HDL-cholesterol, but not with decreased triglycerides. Eichelmann et al., ${ }^{8}$ observed that plant-based diets are associated with an improvement in obesity-related inflammatory profiles, with reductions in the concentrations of C-reactive protein, interleukin-6, and soluble intercellular adhesion molecule- $1 .{ }^{8}$

\section{Keywords}

Plant-based diet, vegetarian diet, cardiovascular health.
Recently, the European Society of Cardiology highlighted that a shift from a more animal-based to a plant-based dietary pattern may reduce the risk of atherosclerotic cardiovascular disease. ${ }^{3}$ In line with this, the American Heart Association (AHA) published a dietary guidance recommending the consumption of healthy sources of protein, mostly from plants, as soybeans, other beans, lentils, chickpeas, and peas to reduce cardiovascular risk. ${ }^{9}$ In addition, there is a growing concern about the impact of the food system on the environment and climate change. A plant-based dietary pattern is more sustainable as it contributes to the reduction of greenhouse gas emissions. ${ }^{10}$ The AHA also reinforced that the replacement if animal-source foods by plant-based whole foods has additional benefits to planetary health. Conversely, a sustainable dietary pattern is not necessarily associated with a lower cardiovascular risk, since a plant-based diet, high in refined carbohydrate and added sugar, may increase the risk of type 2 diabetes and cardiovascular disease (CVD). ${ }^{9}$

Baden et al.,11 investigated the associations between 12-year changes (from 1986 to 1998) in plant-based diet quality assessed by three indices) - an overall plant-based diet index (PDI), a healthful plant-based diet index (hPDI), and an unhealthful plant-based diet index (uPDI) (score range: 18 to 90) - and subsequent total and cause-specific mortality (from 1998 to 2014). The study concluded that improving plant-based diet quality over a 12-year period was associated with a lower risk of total and cardiovascular mortality, whereas increased consumption of an unhealthful plant-based diet was associated with a higher risk of total and CVD mortality. ${ }^{11}$ In another cohort, it was observed that

Mailing Address: Márcia Regina Simas Torres Klein

Rua São Francisco Xavier, 524 - Pavilhão João Lyra Filho. Postal Code: 20559-900, $12^{\circ}$ andar, Bloco D - Rio de Janeiro, RJ - Brazil. Email: marciarsimas@gmail.com 
improving adherence to overall and healthful plantbased diets was associated with a lower risk of type 2 diabetes, whereas decreased adherence to such diets was associated with a higher risk. ${ }^{12}$

In addition to the known benefits of increased fiber intake, the positive effects of healthy vegetarian and plantbased diets also might be attributed by the modulation of gut microbiota composition. Increasing evidence has shown that different dietary patterns affect the gut microbiota, and differences in this ecosystem between vegetarian and omnivores have been documented. Plant-based diets seem to contribute to greater diversity in gut microbiota, which is associated with lower risk of developing metabolic disorders and CVD. ${ }^{13}$ This positive impact may be due to the higher amount of fermentable fibers, polyphenols and polyunsaturated fatty acids in the diet, that act as prebiotics and selectively stimulate the increase of beneficial species. ${ }^{14}$

In this issue of the Journal, Antoniazzi et al., ${ }^{15}$ in a crosssectional study, compare dietary adequacy, according to the recommendations of the National Cholesterol Education Program (NCEP), between apparently healthy vegetarians and omnivorous men. Several cardiovascular risk markers were significantly lower in vegetarians compared to omnivores, including, body mass index, waist circumference, blood pressure, total cholesterol, LDL cholesterol, triglycerides, apolipoprotein B, fasting glucose, glycated hemoglobin, pulse wave velocity, and carotid intima-media thickness. Vegetarians consumed significantly more dietary fibers, polyunsaturated fats and plant stanols, and significantly less protein, total fat, monounsaturated fat, saturated fat and dietary cholesterol. The NCEP recommendations for saturated fat $(<7 \%$ of total calories), dietary cholesterol $(<200 \mathrm{mg} /$ day) and fiber (20-30g/day) were met, respectively by $77 \%, 95 \%$ and $39 \%$ of vegetarians vs. $48 \%, 43 \%$ and $25 \%$ of omnivores $(\mathrm{p}<0.01)$. All vegetarians and omnivores consumed monounsaturated and polyunsaturated fatty acids within NCEP recommendations. Logistic regression analysis showed that, compared with omnivorous diets, vegetarian dietary patterns were associated $(\mathrm{p}<0.05)$ with an adequate intake of saturated fat and dietary cholesterol, even after adjustment for energy intake and age. The authors concluded that vegetarians were more likely to consume saturated fat, cholesterol, and fibers according to NCEP recommendations, which may contribute to lower levels of cardiovascular risk markers.

In summary, the findings of the study conducted by Antoniazzi et al., ${ }^{15}$ are in line with recent evidence suggesting beneficial effects of plant-based diets, and highlight that achieving nutritional recommendations for CVD prevention may be easier for vegetarians compared to omnivores.

\section{References}

1. Kim H, Caulfield LE, Garcia-Larsen V, Steffen LM, Coresh J, Rebholz CM. Plant-Based Diets Are Associated With a Lower Risk of Incident Cardiovascular Disease, Cardiovascular Disease Mortality, and All-Cause Mortality in a General Population of Middle-Aged Adults. J Am Heart Assoc. 2019 Aug 20;8(16):e012865. doi: 10.1161/JAHA.119.012865.

2. Glenn AJ, Lo K, Jenkins DJA, Boucher BA, Hanley AJ, Kendall CWC, et al. Relationship Between a Plant-Based Dietary Portfolio and Risk of Cardiovascular Disease: Findings From the Women's Health Initiative Prospective Cohort Study. J Am Heart Assoc. 2021 Aug 17;10(16):e021515. doi: 10.1161/JAHA.121.021515.

3. Visseren FLJ, Mach F, Smulders YM, Carballo D, Koskinas KC, Bäck M, et al. 2021 ESC Guidelines on cardiovascular disease prevention in clinical practice. Eur Heart J. 2021 Sep 7;42(34):3227-337. doi: 10.1093/ eurhearti/ehab484

4. Choi Y, Larson N, Steffen LM, Schreiner PJ, Gallaher DD, Duprez DA, et al. Plant-Centered Diet and Risk of Incident Cardiovascular Disease During Young to Middle Adulthood. J Am Heart Assoc. 2021 Aug 17;10(16):e020718. doi: 10.1161/JAHA.120.020718.

5. Satija A, Hu FB. Plant-based diets and cardiovascular health. Trends Cardiovasc Med. 2018 Oct;28(7):437-41. doi: 10.1016/j.tcm.2018.02.004.

6. Dinu M, Abbate R, Gensini GF, Casini A, Sofi F. Vegetarian, vegan diets and multiple health outcomes: A systematic review with meta-analysis of observational studies. Crit Rev Food Sci Nutr. 2017 Nov 22;57(17):36409. doi: 10.1080/10408398.2016.1138447.

7. Yokoyama Y, Levin SM, Barnard ND. Association between plant-based diets and plasma lipids: a systematic review and meta-analysis. Nutr Rev. 2017 Sep 1;75(9):683-98. doi: 10.1093/nutrit/nux030.

8. Eichelmann F, Schwingshackl L, Fedirko V, Aleksandrova K. Effect of plant-based diets on obesity-related inflammatory profiles: a systematic review and meta-analysis of intervention trials. Obes Rev. 2016 Nov;17(11):1067-79. doi: 10.1111/obr.12439.

9. Lichtenstein AH, Appel LJ, Vadiveloo M, Hu FB, Kris-Etherton PM, Rebholz CM, et al. 2021 Dietary Guidance to Improve Cardiovascular Health: A Scientific Statement From the American Heart Association. Circulation. 2021 Nov 2;CIR0000000000001031. doi: 10.1161/ CIR.0000000000001031.

10. Swinburn BA, Kraak VI, Allender S, Atkins VI, Baker PI, Bogard JR, et al. The Global Syndemic of Obesity, Undernutrition, and Climate Change: The Lancet Commission report. Lancet. 2019 Feb 23;393(10173):791846. doi: 10.1016/S0140-6736(18)32822-8.

11. Baden MY, Liu G, Satija A, Li Y, Sun Q, Fung TT, et al. Changes in Plant-Based Diet Quality and Total and Cause-Specific Mortality. Circulation. 2019 Sep 17;140(12):979-91. doi: 10.1161/CIRCULATIONAHA.119.041014.

12. Chen Z, Drouin-Chartier J, Li Y, Baden MY, Manson JE, Willett WC,et al. Changes in Plant-Based Diet Indices and Subsequent Risk of Type 2 Diabetes in Women and Men: Three U.S. Prospective Cohorts. Diabetes Care. 2021 Mar; 44(3): 663-71. doi.org/10.2337/dc20-1636. 
13. Tang WHW, Bäckhed F, Landmesser U, Hazen SL. Intestinal Microbiota in Cardiovascular Health and Disease: JACC State-of-theArt Review. J Am Coll Cardiol. 2019;73(16):2089-105. doi:10.1016/j. jacc.2019.03.024

14. Gibson GR, Hutkins R, Sanders ME, Prescott SL, Reimer RA, Salminen SJ, et al. Expert consensus document: The International Scientific Association for Probiotics and Prebiotics (ISAPP) consensus statement on the definition and scope of prebiotics. Nat Rev Gastroenterol Hepatol. 2017 Aug;14(8):491-502. doi: 10.1038/nrgastro.2017.75.

15. Antoniazzi L, Acosta-Navarro J, Oki AM, Bonfim MC, Gaspar MCA Better Adequacy of Food Intake According to Dietary Recommendations of National Cholesterol Education Program in Vegetarian Compared to Omnivorous Men. Int J Cardiovasc Sci. 2022; 35(1):1-10. Doi: https://doi. org/10.36660/ ijcs.20200258. 